\title{
From Actuarial to Financial Valuation Principles
}

\author{
Martin Schweizer \\ Technische Universität Berlin \\ Fachbereich Mathematik, MA 7-4 \\ Straße des 17. Juni 136 \\ $D-10623$ Berlin \\ Germany
}

\begin{abstract}
A valuation principle is a mapping that assigns a number (value) to a random variable (payoff). This paper constructs a transformation on valuation principles by embedding them in a financial environment. Given an a priori valuation rule $u$, we define the associated a posteriori valuation rule $h$ by an indifference argument: The $u$-value of optimally investing in the financial market alone should equal the $u$-value of first selling the payoff at its $h$-value and then choosing an optimal investment strategy inclusive of the payoff. In an $L^{2}$-framework, we explicitly construct in this way the financial transforms of the variance principle and the standard deviation principle.
\end{abstract}

Key words: finance, insurance, variance principle, standard deviation principle, varianceoptimal martingale measure

1991 Mathematics Subject Classification: 62P05, 91B28

JEL Classification Numbers: G10

(Insurance: Mathematics \& Economics 28 (2001), 31-47)

This version: 25.07 .2000 


\section{Introduction}

In recent years, there has been an ever increasing trend to bring finance and insurance closer together. The motivation for the present paper could almost be quoted from Hans Bühlmann (1997) who in this context also coined the term "actuary of the third kind" in Bühlmann (1987): “ . . finance and insurance mathematics should be presented to today's students as one discipline". In accordance with this, our starting point is the observation that the valuation of random amounts is an important topic in both actuarial and financial mathematics and has been studied extensively in both fields. Almost any textbook includes a treatment under headings like premium principles or derivative pricing. We attempt to bring these approaches together by embedding an actuarial valuation principle in a financial environment.

The basic idea is simple. We begin with an a priori valuation rule which assigns a number ("premium", "price") to any random payoff from a suitable class. Typically, this rule is given or motivated by an actuarial premium principle. But the payoffs we consider do not exist in a vacuum; they are surrounded by a financial environment described by the outcomes of trades available to participants in a financial market. Such trades can be used to reduce the risk one has contracted by the sale or purchase of some random amount like an insurance claim or a financial obligation. To value a given payoff in this environment, we compare two procedures. One is to ignore the payoff completely and simply trade on the financial market in a subjectively optimal way. More precisely, one tries to obtain via trading from a given initial capital a final outcome with maximal value, where the value is computed according to the given a priori rule. Alternatively, one can first sell the payoff under consideration to increase one's initial capital and then look for a trade whose resulting net final outcome (trading outcome minus payoff) has again maximal value. The selling price for the payoff is then defined implicitly by equating these two maximal values; it thus compensates for the payoff since one becomes indifferent between optimal trading alone and the combination of selling and optimal trading inclusive of the payoff. The resulting a posteriori valuation is called the financial transform of the a priori valuation rule.

Of course, this abstract program is too general to be feasible. We therefore specialize the financial environment to a frictionless market modelled by a linear subspace $\mathcal{G}$ of $L^{2}$ with a riskless asset $B$. We consider two specific examples of actuarial valuation principles and explicitly determine their financial transforms. As a whole, the paper is a joint venture between finance and insurance. Finance makes explicit the transformation mechanism and insurance provides the input on which the mechanism can operate. In particular, an actuarial justification for the choice of one particular a priori valuation could via this approach lead to a foundation for pricing in an incomplete financial market.

An alternative economic approach to price insurance contracts has recently been suggested by Kliger/Levikson (1998). Their idea is to determine the premium for an insurance product by maximizing some form of expected profit that involves in particular a specific type 
of insolvency costs. However, their method does not include any genuine financial component and thus is rather different from what we propose here.

In some form or other, most of the ideas in our program have appeared before. Using an indifference argument to implicitly determine a valuation is a standard approach from economics; it has recently been taken up again in a number of papers on option pricing under transaction costs. Pricing and hedging with a mean-variance utility has been studied by Mercurio (1996) in a special case of our framework for $\mathcal{G}$. His paper in fact provided the main single source of motivation for our work. Replacing the variance by the standard deviation has been suggested by Aurell/Życzkowski (1996) in the very special case where the financial environment is generated by a trinomial model for stock prices. The main contribution of the present paper is that it combines all these isolated ideas into a unified framework and explicitly points out that this induces a transformation on the set of valuation rules. It would be very interesting to see other examples of financial transforms of particular valuation principles.

The paper is organized as follows. Section 1 explains the financial background $(\mathcal{G}, B)$, introduces a no-arbitrage type condition and presents some of its implications. In particular, we obtain a decomposition of any random payoff into an attainable part and a non-hedgeable part. The former is riskless and the latter contains all the financial riskiness of the payoff. Section 2 describes a change of measure required for working with discounted payoffs under general interest rates and defines the important concept of the $B$-variance-optimal signed $(\mathcal{G}, B)$-martingale measure $\widetilde{P}$. Section 3 gives a more formal description of the basic idea and determines the financial transform of the actuarial variance principle. This turns out to have a very intuitive structure: It is again a variance principle, but the expectation is taken under $\widetilde{P}$ and the variance component (risk-loading) is based only on the non-hedgeable part of the payoff. A similar result is obtained in section 4 where we find the financial transform of the actuarial standard deviation principle. The final section 5 contains two basic examples and an extension.

\section{Approximate profits and projections in $L^{2}$}

This section presents the abstract financial background and some of its basic properties. Let $(\Omega, \mathcal{F}, P)$ be a probability space and $L^{2}=L^{2}(\Omega, \mathcal{F}, P)$ the space of all square-integrable real random variables with scalar product $(Y, Z)=E[Y Z]$ and norm $\|Y\|=\sqrt{E\left[Y^{2}\right]}$. For any subset $\mathcal{Y}$ of $L^{2}$, we denote by $\mathcal{Y}^{\perp}:=\left\{Z \in L^{2} \mid(Z, Y)=0\right.$ for all $\left.Y \in \mathcal{Y}\right\}$ the orthogonal complement and by $\overline{\mathcal{Y}}$ the closure of $\mathcal{Y}$ in $L^{2}$. Fix $B \in L^{2}$ with $B>0 P$-a.s., let $\mathcal{G}$ be a fixed subset of $L^{2}$ and set $\mathcal{A}:=\mathbb{R} B+\mathcal{G}$. We denote by $\pi$ the projection in $L^{2}$ on the closed linear subspace $\mathcal{G}^{\perp}$.

The pair $(\mathcal{G}, B)$ represents the financial environment in which the subsequent considerations take place. An element $g$ of $\mathcal{G}$ models the total gains from trade resulting from a 
self-financing trading strategy with initial capital 0 , and $B$ is interpreted as the final value of some savings account with initial value 1 . The strict positivity of $B$ means that a unit initial investment always leaves one with some money at the end; if one insists on nonnegative interest rates, one should of course strengthen this to $B \geq 1 P$-a.s. An important special case is $B \equiv 1$; this corresponds to expressing everything in discounted quantities. The set $\mathcal{A}$ is the space of those random payoffs which are strictly attainable in the sense that they can be obtained as the final wealth of some trading strategy with some initial capital. Later on, we shall assume that $\mathcal{G}$ is a linear subspace of $L^{2}$; this corresponds to a financial market without frictions like transaction costs, constraints or other restrictions on strategies. Squareintegrability is imposed to obtain a nice Hilbert space structure and because we want means and variances to exist.

Example. Let $\mathcal{T} \subseteq \mathbb{R}_{+}$be a time index set and $X=\left(X_{t}\right)_{t \in \mathcal{T}}$ an $\mathbb{R}^{d_{\text {-valued }}}$ semimartingale with respect to $P$ and a filtration $\mathbb{F}=\left(\mathcal{F}_{t}\right)_{t \in \mathcal{T}}$ on $(\Omega, \mathcal{F})$. Let $\Theta$ be the space of all $\mathbb{R}^{d}$ valued $\mathbb{F}$-predictable $X$-integrable processes $\vartheta=\left(\vartheta_{t}\right)_{t \in \mathcal{T}}$ such that the stochastic integral process $G(\vartheta):=\int \vartheta d X$ is in the space $\mathcal{S}^{2}$ of semimartingales. Then we could take $B \equiv 1$ and $\mathcal{G}:=G_{T}(\Theta)$, where $T:=\sup \mathcal{T}$ is the time horizon of our economy. In this example, $X$ models the discounted price evolution of $d$ risky assets and each $\vartheta \in \Theta$ can be interpreted as a selffinancing dynamic portfolio strategy so that $G_{T}(\vartheta)$ describes the total gains that result from trading according to $\vartheta$. For a continuous-time model where $\mathcal{T}=[0, T]$ for some $T \in(0, \infty]$, the space $G_{T}(\Theta)$ has been studied by Delbaen/Monat/Schachermayer/Schweizer/Stricker (1997) and Choulli/Krawczyk/Stricker (1998, 1999), among others. For $d=1$ and $\mathcal{T}=$ $\{0,1, \ldots, T\}$ with $T \in \mathbb{N}$, Schweizer (1995) has studied the projection in $L^{2}$ on $G_{T}(\Theta)$, and Mercurio (1996) has introduced and computed mean-variance utility prices under an additional (restrictive) condition on $X$. Also in discrete time, Aurell/Życzkowski (1996) have examined mean-standard-deviation utility prices for a very special process $X$. The results of Mercurio (1996) and Aurell/Życzkowski (1996) have been generalized by Gharagozlou (1997) and Schweizer (1999).

Definition. We say that $\mathcal{G}$ admits no approximate profits in $L^{2}$ if $\overline{\mathcal{G}}$ does not contain $B$.

With the preceding interpretations, this notion is very intuitive: It says that one cannot approximate (in the $L^{2}$-sense) the riskless payoff $B$ by a self-financing strategy with initial wealth 0 . This is one way to impose a no-arbitrage condition on the financial environment; loosely speaking, it should be impossible to turn nothing into something without incurring costs. For the case where $B \equiv 1$ and $\mathcal{G}$ consists of elementary stochastic integrals with respect to a given square-integrable stochastic process, a very similar condition has been studied by Stricker (1990). 
Lemma 1. If $\mathcal{G}$ is a linear subspace of $L^{2}$, the following assertions are equivalent:

1) $\mathcal{G}$ admits no approximate profits in $L^{2}$.

2) $\pi(B)$ is not $P$-a.s. identically 0 .

3) $E[B \pi(B)]>0$.

4) $\mathcal{G}^{\perp} \cap(\mathbb{R B}+\overline{\mathcal{G}}) \neq\{0\}$.

5) There is some $Z$ in $\mathcal{G}^{\perp} \cap(\mathbb{R} B+\overline{\mathcal{G}})$ with $(B, Z)>0$.

Proof. Since $\mathcal{G}$ is a linear subspace of $L^{2}, \mathcal{G}^{\perp \perp}=\overline{\mathcal{G}}$. Thus $L^{2}=\mathcal{G}^{\perp} \oplus \overline{\mathcal{G}}$ and so the riskless payoff $B$ has the orthogonal decomposition

$$
B=\pi(B)+(B-\pi(B)) .
$$

In particular, $\pi(B) \in \mathcal{G}^{\perp} \cap(\mathbb{R} B+\overline{\mathcal{G}})$ and

$$
E[B \pi(B)]=E\left[(\pi(B))^{2}\right] \geq 0 .
$$

This shows that 1) -4) are all equivalent and imply 5) with $Z=\pi(B)$. As 5) clearly implies 4), the proof is complete.

\section{q.e.d.}

Actually, it is easy to show that linearity of $\mathcal{G}$ implies that $\mathcal{G}^{\perp} \cap(\mathbb{R B}+\overline{\mathcal{G}})=\pi(\mathbb{R} B)$, but we shall not use this in the sequel. The second part of the next result will not be used either, but may be useful for applications.

Lemma 2. Let $\mathcal{G}$ be a linear subspace of $L^{2}$ admitting no approximate profits in $L^{2}$. Then:

1) $\mathcal{A}^{\perp \perp}=\overline{\mathcal{A}}=\mathbb{R} B+\overline{\mathcal{G}}=\mathbb{R} B+\mathcal{G}^{\perp \perp}$.

2) $\mathcal{A}$ is closed in $L^{2}$ if and only if $\mathcal{G}$ is closed in $L^{2}$.

Proof. 1) Since $\mathcal{A}$ and $\mathcal{G}$ are both linear subspaces, the first and third equalities are clear without further assumptions. Any $g \in \overline{\mathcal{G}}$ is the limit in $L^{2}$ of a sequence $\left(g_{n}\right)$ in $\mathcal{G}$; hence $c B+g_{n}=a_{n}$ is a Cauchy sequence in $\mathcal{A}$ and thus converges in $L^{2}$ to a limit $a \in \overline{\mathcal{A}}$ so that $c B+g=a \in \overline{\mathcal{A}}$. This gives the inclusion " $\supseteq$ " for the middle equality. For the converse, we use the assumption that $\mathcal{G}$ admits no approximate profits in $L^{2}$ to obtain from Lemma 1 an element $Z \in \mathcal{G}^{\perp} \cap(\mathbb{R} B+\overline{\mathcal{G}})$ with $(B, Z)>0$. For any $a \in \overline{\mathcal{A}}$, there is a sequence $a_{n}=c_{n} B+g_{n}$ in $\mathcal{A}$ converging to $a$ in $L^{2}$. Since $c_{n} B+g_{n} \in \mathbb{R} B+\mathcal{G}$ for all $n$, we conclude that

$$
\left(a_{n}, Z\right)=\left(c_{n} B+g_{n}, Z\right)=\left(c_{n} B, Z\right)=c_{n}(B, Z)
$$


converges in $\mathbb{R}$ to $(a, Z)$ and since $(B, Z)>0,\left(c_{n}\right)$ converges to $c=\frac{(a, Z)}{(B, Z)}$. Therefore $g_{n}=a_{n}-c_{n} B$ converges in $L^{2}$ to $g:=a-c B$ and since this limit is in $\overline{\mathcal{G}}$, we have $a=$ $c B+g \in \mathbb{R} B+\overline{\mathcal{G}}$ which proves the inclusion " $\subseteq$ ".

2) The "if" part is immediate from 1). Conversely, let $\left(g_{n}\right)$ be any sequence in $\mathcal{G}$ converging in $L^{2}$ to some $g_{\infty}$ which is in $\overline{\mathcal{A}}$ since $\mathcal{G} \subseteq \mathcal{A}$. If $\mathcal{A}$ is closed in $L^{2}$, we obtain $g_{\infty} \in \mathcal{A}$, hence $g_{\infty}=c B+g$ for some $c \in \mathbb{R}$ and $g \in \mathcal{G}$. Because $\mathcal{G}$ admits no approximate profits in $L^{2}$, we can choose $Z$ as in Lemma 1 to obtain

$$
c(B, Z)=(c B+g, Z)=\left(g_{\infty}, Z\right)=\lim _{n \rightarrow \infty}\left(g_{n}, Z\right)=0
$$

since $g_{n} \in \mathcal{G}$ and $Z \in \mathcal{G}^{\perp}$. But since $(B, Z)>0$, we conclude that $c=0$ so that $g_{\infty}=g \in \mathcal{G}$ and this proves the "only if" part.

q.e.d.

The next result provides an elementary but important decomposition for arbitrary payoffs in $L^{2}$. Its interpretation is deferred until the end of section 2 .

Corollary 3. Let $\mathcal{G}$ be a linear subspace of $L^{2}$ admitting no approximate profits in $L^{2}$. Then every $H \in L^{2}$ has a unique decomposition as

$$
H=c^{H} B+g^{H}+N^{H} \quad \text { with } c^{H} \in \mathbb{R}, g^{H} \in \overline{\mathcal{G}} \text { and } N^{H} \in \overline{\mathcal{A}}^{\perp} \text {. }
$$

In particular, we have $E\left[B N^{H}\right]=0$ and $E\left[N^{H} g\right]=0$ for all $g \in \overline{\mathcal{G}}$.

Proof. Writing $L^{2}=\overline{\mathcal{A}} \oplus \overline{\mathcal{A}}^{\perp}$ yields $H=a^{H}+N^{H}$ with unique elements $a^{H}=c^{H} B+g^{H} \in \overline{\mathcal{A}}$ and $N^{H} \in \overline{\mathcal{A}}^{\perp}$. If $a^{H}=c B+g$ for some $c \in \mathbb{R}$ and $g \in \overline{\mathcal{G}}$ and if $Z$ is as in Lemma 1, we obtain $c(B, Z)=\left(a^{H}, Z\right)=c^{H}(B, Z)$ which proves the uniqueness of the decomposition (1.1).

q.e.d.

Consider now the optimization problem

$$
\text { Minimize } E\left[(H-c B-g)^{2}\right] \text { over all } c \in \mathbb{R} \text { and all } g \in \mathcal{G}
$$

for a fixed $H \in L^{2}$. Since $\mathcal{A}=\mathbb{R} B+\mathcal{G}$, this can equivalently be rewritten as

$$
\text { Minimize }\|H-a\|^{2} \text { over all } a \in \mathcal{A}
$$

and is therefore recognized as the problem of projecting $H$ in $L^{2}$ on $\mathcal{A}$. The minimal distance will later turn out to play an important role. 
Lemma 4. Let $\mathcal{G}$ be a linear subspace of $L^{2}$ admitting no approximate profits in $L^{2}$. For every fixed $H \in L^{2}$, we then have in terms of the decomposition (1.1) that

$$
J_{0}:=\inf _{(c, g) \in \mathbb{R} \times \mathcal{G}} E\left[(H-c B-g)^{2}\right]=\inf _{a \in \mathcal{A}}\|H-a\|^{2}=E\left[\left(N^{H}\right)^{2}\right] .
$$

If $g^{H}$ is in $\mathcal{G}$, then the solution of (1.2) or (1.3) is given by $\left(c^{H}, g^{H}\right) \in \mathbb{R} \times \mathcal{G}$. In particular, this is always the case if $\mathcal{G}$ is closed in $L^{2}$.

Proof. Clearly, we have $J_{0}=\inf _{a \in \mathcal{A}}\|H-a\|^{2}=\inf _{a \in \overline{\mathcal{A}}}\|H-a\|^{2}$. By Corollary 3, the projection of $H$ in $L^{2}$ on $\overline{\mathcal{A}}$ is $c^{H} B+g^{H}$ and so

$$
J_{0}=\left\|H-c^{H} B-g^{H}\right\|^{2}=\left\|N^{H}\right\|^{2}=E\left[\left(N^{H}\right)^{2}\right] .
$$

The remaining assertions are clear.

q.e.d.

\section{Choosing the measure}

In this section, we introduce some additional measures on $(\Omega, \mathcal{F})$ for two reasons. Firstly, we can then give alternative expressions for the quantity $J_{0}$ in Lemma 4 and the constant $c^{H}$ in the decomposition (1.1) of $H$. More importantly, however, one of these measures is also needed in the formulation of our a priori valuation rules in the next section.

To motivate the next definition, we first reconsider the problem (1.2) of minimizing $E\left[(H-c B-g)^{2}\right]$ over all $c \in \mathbb{R}$ and $g \in \mathcal{G}$. Think of $H$ as some random payoff to be made at the final date in our environment. If we choose some initial capital $c$ and some selffinancing strategy represented by some $g \in \mathcal{G}$, our final wealth will be $c B+g$ and thus leave us with a net loss of $H-c B-g$ at the end. Hence (1.2) is just the problem of minimizing the expected squared net loss by choice of an appropriate strategy $(c, g)$.

In this formulation, all payoffs are measured in the originally given monetary units. If we want to re-express this in units of our riskless asset $B$, we have to divide all final date payments by $B$; this is also called discounting with respect to $B$. In these new units, we can then write (1.2) as minimizing

$$
E\left[B^{2}\left(\frac{H}{B}-c-\frac{g}{B}\right)^{2}\right] \quad \text { over }(c, g) \in \mathbb{R} \times \mathcal{G}
$$

or equivalently as

$$
\text { Minimize } E^{B}\left[\left(\frac{H}{B}-c-\frac{g}{B}\right)^{2}\right] \text { over all } c \in \mathbb{R} \text { and all } g \in \mathcal{G}
$$


where the new probability measure $P^{B} \approx P$ is defined by

$$
\frac{d P^{B}}{d P}:=\frac{B^{2}}{E\left[B^{2}\right]}
$$

Here and in the sequel, all expectations and variances without indices refer to $P$. It is clear that a random variable $H$ is in $L^{2}=L^{2}(P)$ if and only if $\frac{H}{B}$ is in $L^{2}\left(P^{B}\right)$ and thus $P^{B}$ is the natural measure for working with $B$-discounted quantities.

We point out that $P^{B}$ only comes in explicitly if we have "stochastic interest rates" in the sense that $B$ is not deterministic. If $B=b P$-a.s. for some constant $b>0$, then $(2.1)$ immediately yields that $P^{B}=P$. In order to work with discounted quantities in a general framework, we focus henceforth on the measure $P^{B}$. The next result collects some simple properties for later use; superscripts ${ }^{B}$ refer to $P^{B}$.

Lemma 5. If $H \in L^{2}$ has a decomposition $H=c^{H} B+g^{H}+N^{H}$ as in (1.1), then $E^{B}\left[\frac{N^{H}}{B}\right]=0$ and $\operatorname{Cov}^{B}\left(\frac{g}{B}, \frac{N^{H}}{B}\right)=0$ for all $g \in \overline{\mathcal{G}}$. This means that under $P^{B}, \frac{N^{H}}{B}$ has zero mean and is uncorrelated with the space $\frac{1}{B} \overline{\mathcal{G}}$ of $B$-discounted gains.

Proof. From the definition of $P^{B}$, we obtain $E^{B}\left[\frac{N^{H}}{B}\right]=\frac{1}{E\left[B^{2}\right]} E\left[B N^{H}\right]=0$ by (1.1). Hence we have for all $g \in \overline{\mathcal{G}}$ again by $(1.1)$

$$
\operatorname{Cov}^{B}\left(\frac{g}{B}, \frac{N^{H}}{B}\right)=E^{B}\left[\frac{g}{B} \frac{N^{H}}{B}\right]=\frac{1}{E\left[B^{2}\right]} E\left[g N^{H}\right]=0 .
$$

The next concept has been introduced by Schweizer (1996) for $B \equiv 1$; the extension to positive $B$ follows Gouriéroux/Laurent/Pham (1998).

Definition. A signed $(\mathcal{G}, B)$-martingale measure is a signed measure $Q$ on $(\Omega, \mathcal{F})$ with $Q[\Omega]=1, Q \ll P$ with $\frac{1}{B} \frac{d Q}{d P} \in L^{2}$ and

$$
E_{Q}\left[\frac{g}{B}\right]=\left(\frac{1}{B} \frac{d Q}{d P}, g\right)=0 \quad \text { for all } g \in \mathcal{G}
$$

A signed $(\mathcal{G}, B)$-martingale measure $\widetilde{P}$ is called $B$-variance-optimal if

$$
\left\|\frac{1}{B} \frac{d \widetilde{P}}{d P}\right\| \leq\left\|\frac{1}{B} \frac{d Q}{d P}\right\| \quad \text { for all signed }(\mathcal{G}, B) \text {-martingale measures } Q
$$

(To indicate the dependence on $B$, we could write $\widetilde{P}^{B}$ instead of $\widetilde{P}$, but as $B$ is fixed throughout, this would only clutter up the notation.) 
Remarks. 1) For every signed $(\mathcal{G}, B)$-martingale measure $Q, E^{B}\left[\frac{d Q}{d P^{B}}\right]=Q[\Omega]=1$. Since $\frac{d Q}{d P}=\frac{d Q}{d P^{B}} \frac{B^{2}}{E\left[B^{2}\right]}$ by the definition of $P^{B}$, we obtain

$$
\left\|\frac{1}{B} \frac{d Q}{d P}\right\|^{2}=\frac{1}{E\left[B^{2}\right]} E^{B}\left[\left(\frac{d Q}{d P^{B}}\right)^{2}\right]=\frac{1}{E\left[B^{2}\right]}\left(\operatorname{Var}^{B}\left[\frac{d Q}{d P^{B}}\right]+1\right) .
$$

Thus $\widetilde{P}$ is $B$-variance-optimal if and only if its $B$-discounted density with respect to $P^{B}$ has minimal $P^{B}$-variance among all signed $(\mathcal{G}, B)$-martingale measures $Q$; hence the terminology.

2) As already pointed out in Delbaen/Schachermayer (1996), one has to consider signed measures because without additional assumptions, $\widetilde{P}$ does turn out to become negative on some sets. This reflects the fact that a quadratic "utility function" (which underlies the idea of a mean-variance criterion) is not increasing.

Lemma 6. Let $\mathcal{G}$ be a linear subspace of $L^{2}$. The $B$-variance-optimal signed $(\mathcal{G}, B)$-martingale measure $\widetilde{P}$ exists if and only if $\mathcal{G}$ admits no approximate profits in $L^{2}$. In that case, $\widetilde{P}$ is unique and given by

$$
\frac{d \widetilde{P}}{d P}=\frac{B \pi(B)}{E[B \pi(B)]} \quad \text { or } \quad \frac{d \widetilde{P}}{d P^{B}}=\frac{\frac{\pi(B)}{B}}{E^{B}\left[\frac{\pi(B)}{B}\right]}
$$

Proof. For $B \equiv 1$, this can be found in Delbaen/Schachermayer (1996) or Schweizer (1996). The argument for general $B>0$ is quite similar, but we include it here for completeness. If $Q$ is a signed $(\mathcal{G}, B)$-martingale measure, then $\frac{1}{B} \frac{d Q}{d P} \in \mathcal{G}^{\perp}$ by definition and so

$$
1=Q[\Omega]=\left(\frac{1}{B} \frac{d Q}{d P}, B\right)=\left(\frac{1}{B} \frac{d Q}{d P}, \pi(B)\right)
$$

This shows that the set of signed $(\mathcal{G}, B)$-martingale measures is nonempty if and only if $\pi(B) \not \equiv 0$, hence by Lemma 1 if and only if $\mathcal{G}$ admits no approximate profits in $L^{2}$. Since the set of all $B$-discounted densities $\frac{1}{B} \frac{d Q}{d P}$ of signed $(\mathcal{G}, B)$-martingale measures $Q$ is convex and closed in $L^{2}$, it is clear that the $B$-variance-optimal signed $(\mathcal{G}, B)$-martingale measure then exists and is unique. To prove $(2.2)$, we first note that $\frac{B \pi(B)}{E[B \pi(B)]}$ is well-defined by Lemma 1 since $\mathcal{G}$ admits no approximate profits in $L^{2}$ and so $\widetilde{P}$ defined by the first equation in $(2.2)$ is a signed $(\mathcal{G}, B)$-martingale measure. Moreover, $\pi(B)$ is in $\mathbb{R} B+\overline{\mathcal{G}}$ according to the proof of Lemma 1 , hence of the form $c B+g$ for some $c \in \mathbb{R}$ and $g \in \overline{\mathcal{G}}$, and so

$$
\left(\frac{1}{B} \frac{d Q}{d P}, \frac{1}{B} \frac{d \widetilde{P}}{d P}\right)=\frac{1}{E[B \pi(B)]}\left(\frac{1}{B} \frac{d Q}{d P}, \pi(B)\right)=\frac{c}{E[B \pi(B)]}
$$


is constant over all signed $(\mathcal{G}, B)$-martingale measures $Q$. If $Q$ is now an arbitrary signed $(\mathcal{G}, B)$-martingale measure, then so is $R:=2 Q-\widetilde{P}$, and $Q=\widetilde{P}+\frac{R-\widetilde{P}}{2}$. Hence we obtain

$$
\left\|\frac{1}{B} \frac{d Q}{d P}\right\|^{2}=\left\|\frac{1}{B} \frac{d \widetilde{P}}{d P}\right\|^{2}+\frac{1}{4}\left\|\frac{1}{B} \frac{d(R-\widetilde{P})}{d P}\right\|^{2} \geq\left\|\frac{1}{B} \frac{d \widetilde{P}}{d P}\right\|^{2}
$$

because the mixed term disappears thanks to (2.3). This proves that $\widetilde{P}$ defined by the first expression in (2.2) is indeed $B$-variance-optimal. The alternative second expression follows if we use the first half of $(2.2)$ and the definition of $P^{B}$ to obtain

$$
\frac{d \widetilde{P}}{d P^{B}}=\frac{d \widetilde{P}}{d P} \frac{d P}{d P^{B}}=\frac{\pi(B) E\left[B^{2}\right]}{B E[B \pi(B)]}=\frac{\frac{\pi(B)}{B}}{E^{B}\left[\frac{\pi(B)}{B}\right]} .
$$

q.e.d.

For future reference, we compute the minimal variance $\operatorname{Var}^{B}\left[\frac{d \widetilde{P}}{d P^{B}}\right]$ explicitly in terms of $P, B$ and $\pi(B)$. We first observe that

$$
E^{B}\left[\left(\frac{\pi(B)}{B}\right)^{2}\right]=\frac{1}{E\left[B^{2}\right]} E\left[(\pi(B))^{2}\right]=\frac{1}{E\left[B^{2}\right]} E[B \pi(B)]=E^{B}\left[\frac{\pi(B)}{B}\right] .
$$

Thanks to (2.2) and the orthogonality of $\pi(B)$ and $B-\pi(B)$ in $L^{2}$, we thus obtain (2.4) $\operatorname{Var}^{B}\left[\frac{d \widetilde{P}}{d P^{B}}\right]=\frac{1}{E^{B}\left[\frac{\pi(B)}{B}\right]}-1=\frac{E^{B}\left[1-\frac{\pi(B)}{B}\right]}{E^{B}\left[\frac{\pi(B)}{B}\right]}=\frac{E[B(B-\pi(B))]}{E[B \pi(B)]}=\frac{\|B-\pi(B)\|^{2}}{\|\pi(B)\|^{2}}$.

Corollary 7. Let $\mathcal{G}$ be a linear subspace of $L^{2}$ admitting no approximate profits in $L^{2}$. For $H \in L^{2}$, consider the representation $H=c^{H} B+g^{H}+N^{H}$ from Corollary 3. Then

$$
c^{H}=\widetilde{E}\left[\frac{H}{B}\right]=\left(\frac{1}{B} \frac{d \widetilde{P}}{d P}, H\right),
$$

where $\widetilde{E}$ denotes expectation with respect to the $B$-variance-optimal signed $(\mathcal{G}, B)$-martingale measure $\widetilde{P}$. Moreover,

$$
J_{0}=\inf _{a \in \mathcal{A}}\|H-a\|^{2}=E\left[B^{2}\right] \operatorname{Var}^{B}\left[\frac{N^{H}}{B}\right] .
$$

Proof. By (2.2) and the proof of Lemma $1, \frac{1}{B} \frac{d \widetilde{P}}{d P}$ is in $\mathcal{G}^{\perp} \cap(\mathbb{R} B+\overline{\mathcal{G}})=\mathcal{G}^{\perp} \cap \overline{\mathcal{A}}$ by Lemma 2. Since $g^{H} \in \overline{\mathcal{G}}$ and $N^{H} \in \overline{\mathcal{A}}^{\perp}$, we thus obtain

$$
\widetilde{E}\left[\frac{H}{B}\right]=\left(\frac{1}{B} \frac{d \widetilde{P}}{d P}, c^{H} B+g^{H}+N^{H}\right)=c^{H} \widetilde{P}[\Omega]=c^{H} .
$$


Moreover, Lemma 4 and Lemma 5 imply that

$$
J_{0}=E\left[\left(N^{H}\right)^{2}\right]=E\left[B^{2}\right] E^{B}\left[\left(\frac{N^{H}}{B}\right)^{2}\right]=E\left[B^{2}\right] \operatorname{Var}^{B}\left[\frac{N^{H}}{B}\right]
$$

q.e.d.

Corollary 7 allows us to give a very intuitive financial interpretation for the decomposition

$$
H=c^{H} B+g^{H}+N^{H}
$$

in Corollary 3. It tells us that $H$ splits naturally into an attainable part $c^{H} B+g^{H} \in \overline{\mathcal{A}}$ and a non-hedgeable part $N^{H}$ which is orthogonal to the space $\overline{\mathcal{A}}$ of attainable payoffs. The constant $c^{H}$ is the initial capital of the attainable part; it can be computed in a simple way as the $\widetilde{P}$-expectation of the $B$-discounted payoff $\frac{H}{B}$. Moreover, $(2.5)$ tells us that the $P^{B}$. variance of the $B$-discounted non-hedgeable part of $H$ is a measure for the intrinsic financial risk of $H$ in terms of approximation in $L^{2}$ by attainable, hence perfectly replicable payoffs.

Remark. In the present generality, one has to be a bit careful about the above interpretation. In fact, it may happen that a nonnegative payoff $H \geq 0$ has $c^{H}<0$ because $\widetilde{P}$ is in general a signed measure. The intuition behind such a situation is that a quadratic "utility" has also a part where one is actually risk-seeking instead of risk-averse and that the values of $H$ lie to a large extent in that part.

\section{The financial variance principle}

This section explains the basic idea behind our approach and shows in a first example how it works. We consider a fixed random variable $H \in L^{2}$ and think of this as a random payoff as in section 2. In a financial context, $H$ could represent the net payoff of some derivative product, e.g., a European call option. In insurance terms, $-H$ should be thought of as a claim amount to be paid by the insurer. Since financial derivatives are often termed contingent claims, it seems appropriate to call $H$ a general claim. How much should we charge or pay if we sell or buy $\gamma$ units of this claim?

This question has a standard answer from classical option pricing theory in the special case where $H$ is strictly attainable in the sense that $H \in \mathcal{A}$. Then we can write $H=c^{H} B+g^{H}$ with $c^{H} \in \mathbb{R}$ and $g^{H} \in \mathcal{G}$, and the price per unit of $H$ must be $c^{H}$ to avoid arbitrage. To understand the idea behind this argument, suppose for instance that $H$ is nonnegative and offered at a price $x<c^{H}$. Then one can buy $\frac{c^{H}}{x}$ units of $H$ and finance this initial outlay of $c^{H}$ with $\left(-c^{H},-g^{H}\right)$, i.e., by borrowing the amount $c^{H}$ and trading according to the strategy 
associated to $-g^{H}$. The terminal payoff from this costless deal is then $\frac{c^{H}}{x} H-c^{H} B-g^{H}=$ $\left(\frac{c^{H}}{x}-1\right) H \geq 0$; thus one has turned nothing into something, but absence of arbitrage postulates that this is impossible. A similar argument applies if one can sell $H$ for more than $c^{H}$. However, this entire reasoning depends crucially on the attainability of $H$ and therefore breaks down in a general incomplete financial market. A typical contingent claim there is not attainable and its price will depend on subjective preferences.

If we think of $-H$ as an insurance risk, there is also a standard approach to its valuation from actuarial mathematics: We simply apply a valuation principle appropriate for our needs. Thus we choose a mapping $u$ from random variables $Y$ into $\mathbb{R}$ and think of $u(Y)$ as value or utility associated to the random amount $Y$. For instance, the classical actuarial variance principle would correspond to $u(Y):=$ (expected value of $Y-A$ times the variance of $Y$ ); the slightly unfamiliar choice of signs is due to our convention that $-H$ and not $H$ corresponds to an insurance claim.

Just applying some $u$ to $H$ is of course one possible way to arrive at a valuation. But from a financial perspective, this is too simplistic because it ignores the trading opportunities represented by $\mathcal{G}$. We therefore use a utility indifference argument to obtain our financial valuation rule as a transform of $u$. This is a well-known general approach in economics and we follow here Mercurio (1996) and Aurell/Życzkowski (1996) who combined this with an $L^{2}$-framework to determine option prices in some special cases. For a general formulation of the basic idea, we start with an initial capital $c \in \mathbb{R}$ and an a priori valuation principle $u$. In order to decide on the price of $\gamma$ units of $H$, we compare the following two alternatives:

(i) Invest optimally into a trading strategy with initial capital $c$ and ignore the possibility of selling $H$. This amounts to maximizing $u(c B+g)$ over all $g \in \mathcal{G}$ and we denote the value of this control problem by

$$
v(c, 0):=\sup _{g \in \mathcal{G}} u(c B+g)
$$

(ii) Sell $\gamma$ units of $H$ for the amount $h(c, \gamma) \in \mathbb{R}$ to increase the initial wealth to $c+h(c, \gamma)$. This can then be invested into a trading strategy and leads to a total final wealth of $(c+h(c, \gamma)) B+g-\gamma H$ since we have to pay out the claims from $H$ at the end. For an optimal investment, we thus have to maximize $u((c+h(c, \gamma)) B+g-\gamma H)$ over all $g \in \mathcal{G}$ and the value of this second control problem is

$$
v(c, \gamma):=\sup _{g \in \mathcal{G}} u((c+h(c, \gamma)) B+g-\gamma H)
$$

Indifference with respect to $u$ prevails if $h(c, \gamma)$ is chosen in such a way that neither of these alternatives is preferred to the other. More formally: 
Definition. We call $h(c, \gamma)$ a u-indifference price for $\gamma$ units of $H$ if $v(c, \gamma)=v(c, 0)$, i.e., if $h(c, \gamma)$ satisfies

$$
\sup _{g \in \mathcal{G}} u((c+h(c, \gamma)) B+g-\gamma H)=\sup _{g \in \mathcal{G}} u(c B+g)
$$

Due to the generality of the preceding approach, not much can be said about the properties of $h(c, \gamma)$ at this stage. Of course, $h(c, 0)=0$ is always a $u$-indifference price for 0 units of $H$, although $h(c, \gamma)$ need not be unique. If $H=c^{H} B+g^{H} \in \mathcal{A}$ is strictly attainable, it is also easily checked that $h(c, \gamma)=\gamma c^{H}$ is a $u$-indifference price whatever $u$ is. Thus our approach is consistent with absence of arbitrage.

Recall now the probability measure $P^{B} \approx P$ introduced in section 2 . As explained there, this is the measure one should use to work with $B$-discounted quantities in an $L^{2}$-context. Our goal is to determine the $u$-indifference prices for the two valuation principles

$$
u_{1}(Y):=E^{B}\left[\frac{Y}{B}\right]-A \operatorname{Var}^{B}\left[\frac{Y}{B}\right]
$$

and

$$
u_{2}(Y):=E^{B}\left[\frac{Y}{B}\right]-A \sqrt{\operatorname{Var}^{B}\left[\frac{Y}{B}\right]}
$$

where $A>0$ is a risk aversion parameter. The corresponding $u$-indifference prices will be denoted by $h_{1,2}(c, \gamma)$ respectively.

Lemma 8. For $u \in\left\{u_{1}, u_{2}\right\}$, the $u$-indifference price $h(c, \gamma)$ for any $H \in L^{2}$ does not depend on $c$ and is for all $\gamma, c \in \mathbb{R}$ given by

$$
h(c, \gamma)=h(\gamma)=\sup _{g \in \mathcal{G}} u(g)-\sup _{g \in \mathcal{G}} u(g-\gamma H)=\sup _{g \in \overline{\mathcal{G}}} u(g)-\sup _{g \in \overline{\mathcal{G}}} u(g-\gamma H)
$$

Proof. Since the variance of a random variable does not change if we add a constant,

$$
\begin{aligned}
u_{1,2}((c+x) B+g-\gamma H) & =E^{B}\left[c+x+\frac{g-\gamma H}{B}\right]-A\left(\operatorname{Var}^{B}\left[c+x+\frac{g-\gamma H}{B}\right]\right)^{\beta_{1,2}} \\
& =c+x+u_{1,2}(g-\gamma H)
\end{aligned}
$$

for any $c, x \in \mathbb{R}$, where $\beta_{1}=1$ and $\beta_{2}=\frac{1}{2}$. This implies that

$$
v(c, \gamma)=\sup _{g \in \mathcal{G}} u((c+h(c, \gamma)) B+g-\gamma H)=c+h(c, \gamma)+\sup _{g \in \mathcal{G}} u(g-\gamma H)
$$


and

$$
v(c, 0)=\sup _{g \in \mathcal{G}} u(c+g)=c+\sup _{g \in \mathcal{G}} u(g)
$$

for $u \in\left\{u_{1}, u_{2}\right\}$. Since $h(c, \gamma)$ is defined by (3.1), we obtain the first and second equalities.

For the third equality, it is obviously enough to show that for any $Y \in L^{2}$, we have

$$
L:=\sup _{g \in \mathcal{G}} u(g+Y)=\sup _{g \in \overline{\mathcal{G}}} u(g+Y)=: R .
$$

Clearly $L \leq R$ and so it only remains to show that $L \geq R$. For any $g \in \overline{\mathcal{G}}$, there is a sequence $\left(g_{n}\right)_{n \in \mathbb{N}}$ in $\mathcal{G}$ converging to $g$ in $L^{2}$. This implies that $E^{B}\left[\frac{g_{n}+Y}{B}\right]=\frac{1}{E\left[B^{2}\right]} E\left[B\left(g_{n}+Y\right)\right]$ converges to $E^{B}\left[\frac{g+Y}{B}\right]$ and that $\left(\left\|g_{n}+Y\right\|\right)_{n \in \mathbb{N}}$ is bounded in $n$. Since

$$
\left|\left\|g_{n}+Y\right\|^{2}-\|g+Y\|^{2}\right| \leq\left\|g_{n}-g\right\|\left(\sup _{n \in \mathbb{N}}\left\|g_{n}+Y\right\|+\|g+Y\|\right)
$$

and

$$
\begin{aligned}
& \left|\left(E\left[B\left(g_{n}+Y\right)\right]\right)^{2}-(E[B(g+Y)])^{2}\right| \\
& \leq\left|E\left[B\left(g_{n}-g\right)\right]\right|\left(\sup _{n \in \mathbb{N}}\left|E\left[B\left(g_{n}+Y\right)\right]\right|+|E[B(g+Y)]|\right) \\
& \leq\|B\|^{2}\left\|g_{n}-g\right\|\left(\sup _{n \in \mathbb{N}}\left\|g_{n}+Y\right\|+\|g+Y\|\right),
\end{aligned}
$$

we conclude that

$$
\begin{aligned}
& \left|\operatorname{Var}^{B}\left[\frac{g_{n}+Y}{B}\right]-\operatorname{Var}^{B}\left[\frac{g+Y}{B}\right]\right| \\
& \leq \frac{1}{E\left[B^{2}\right]}\left|E\left[\left(g_{n}+Y\right)^{2}\right]-E\left[(g+Y)^{2}\right]\right|+\frac{1}{\left(E\left[B^{2}\right]\right)^{2}}\left|\left(E\left[B\left(g_{n}+Y\right)\right]\right)^{2}-(E[B(g+Y)])^{2}\right|
\end{aligned}
$$

converges to 0 as $n \rightarrow \infty$. Given $\varepsilon>0$, we thus have $E^{B}\left[\frac{g_{n}+Y}{B}\right] \geq E^{B}\left[\frac{g+Y}{B}\right]-\varepsilon$ and $\operatorname{Var}^{B}\left[\frac{g_{n}+Y}{B}\right] \leq \operatorname{Var}^{B}\left[\frac{g+Y}{B}\right]+\varepsilon$ for $n$ sufficiently large. This implies that

$$
\begin{aligned}
L & \geq u\left(g_{n}+Y\right) \\
& =E^{B}\left[\frac{g_{n}+Y}{B}\right]-A\left(\operatorname{Var}^{B}\left[\frac{g_{n}+Y}{B}\right]\right)^{\beta} \\
& \geq E^{B}\left[\frac{g+Y}{B}\right]-\varepsilon-A\left(\operatorname{Var}^{B}\left[\frac{g+Y}{B}\right]+\varepsilon\right)^{\beta}
\end{aligned}
$$

for $n$ sufficiently large, hence

$$
L \geq E^{B}\left[\frac{g+Y}{B}\right]-A\left(\operatorname{Var}^{B}\left[\frac{g+Y}{B}\right]\right)^{\beta}=u(g+Y)
$$


by letting $\varepsilon$ tend to 0 and since $g \in \overline{\mathcal{G}}$ was arbitrary, we obtain (3.3).

q.e.d.

Theorem 9. Let $\mathcal{G}$ be a linear subspace of $L^{2}$ admitting no approximate profits in $L^{2}$. For any $H \in L^{2}$ and any $\gamma, c \in \mathbb{R}$, the $u_{1}$-indifference price for $\gamma$ units of $H$ is then

$$
h_{1}(c, \gamma)=h_{1}(\gamma)=\gamma c^{H}+A \gamma^{2} \frac{J_{0}}{E\left[B^{2}\right]}=\gamma \widetilde{E}\left[\frac{H}{B}\right]+A \gamma^{2} \operatorname{Var}^{B}\left[\frac{N^{H}}{B}\right] \text {, }
$$

where $\widetilde{E}$ denotes expectation with respect to the $B$-variance-optimal signed $(\mathcal{G}, B)$-martingale measure $\widetilde{P}$.

Proof. By Corollary 3, $H$ can be decomposed as $H=c^{H} B+g^{H}+N^{H}$ as in (1.1) so that

$$
u_{1}(g-\gamma H)=u_{1}\left(-\gamma c^{H} B+g-\gamma g^{H}-\gamma N^{H}\right)=-\gamma c^{H}+u_{1}\left(g-\gamma g^{H}-\gamma N^{H}\right) .
$$

Since $\mathcal{G}$ is a linear subspace of $L^{2}$, the mapping $g \mapsto g^{\prime}:=g-\gamma g^{H}$ is a bijection of $\overline{\mathcal{G}}$ into itself for every fixed $H \in L^{2}$ and $\gamma \in \mathbb{R}$ and so Lemma 8 implies that

$$
h_{1}(\gamma)=\sup _{g \in \overline{\mathcal{G}}} u_{1}(g)+\gamma c^{H}-\sup _{g^{\prime} \in \overline{\mathcal{G}}} u_{1}\left(g^{\prime}-\gamma N^{H}\right) .
$$

But $E^{B}\left[\frac{N^{H}}{B}\right]=0$ and $\operatorname{Cov}^{B}\left(\frac{g^{\prime}}{B}, \frac{N^{H}}{B}\right)=0$ for all $g^{\prime} \in \overline{\mathcal{G}}$ by Lemma 5 and so we obtain

$$
\begin{aligned}
u_{1}\left(g^{\prime}-\gamma N^{H}\right) & =E^{B}\left[\frac{g^{\prime}-\gamma N^{H}}{B}\right]-A \operatorname{Var}^{B}\left[\frac{g^{\prime}-\gamma N^{H}}{B}\right] \\
& =E^{B}\left[\frac{g^{\prime}}{B}\right]-A \operatorname{Var}^{B}\left[\frac{g^{\prime}}{B}\right]-A \gamma^{2} \operatorname{Var}^{B}\left[\frac{N^{H}}{B}\right] \\
& =u_{1}\left(g^{\prime}\right)-A \gamma^{2} \operatorname{Var}^{B}\left[\frac{N^{H}}{B}\right] .
\end{aligned}
$$

Combining this with (3.5) yields

$$
h_{1}(\gamma)=\sup _{g \in \overline{\mathcal{G}}} u_{1}(g)+\gamma c^{H}-\sup _{g^{\prime} \in \overline{\mathcal{G}}} u_{1}\left(g^{\prime}\right)+A \gamma^{2} \operatorname{Var}^{B}\left[\frac{N^{H}}{B}\right]
$$

and this together with Corollary 7 implies the assertion.

q.e.d.

The valuation formula (3.4) in Theorem 9 has a number of very attractive features. To see the most striking of these, let us interpret $\pm h_{1}( \pm 1)$ as the buying $(\gamma=-1)$ and selling $(\gamma=+1)$ prices for one unit of $H$ respectively. Then we see from (3.4) that

$$
\pm h_{1}( \pm 1)=\widetilde{E}\left[\frac{H}{B}\right] \pm A \operatorname{Var}^{B}\left[\frac{N^{H}}{B}\right] .
$$


This looks very similar to (3.2), but differs by two important points. First of all, the expectation of the $B$-discounted claim $\frac{H}{B}$ is not taken under the original measure $P^{B}$, but under the $B$-variance-optimal signed $(\mathcal{G}, B)$-martingale measure $\widetilde{P}$. Secondly, the variance component in (3.6) is not based on the entire claim $H$, but only on its non-hedgeable part $N^{H}$. Thus we have to pass from the real-world measure $P^{B}$ to the (appropriate) risk-neutral measure $\widetilde{P}$ for computing expectations, and we do not add or subtract a risk-loading (under $P^{B}$ ) for that part of $H$ which can be hedged away by judicious trading. In view of the perfect analogy to (3.2), the prescription (3.6) could be called the financial variance principle.

If $H$ is attainable in the sense that $H=c^{H} B+g^{H}$ is in $\overline{\mathcal{A}}$, then (3.6) reduces to $\pm h_{1}( \pm 1)=c^{H}$. This is exactly what we expect from the classical arbitrage arguments from option pricing. For a general claim $H$, we obtain a bid-ask spread

$$
h_{1}(+1)+h_{1}(-1)=2 A \operatorname{Var}^{B}\left[\frac{N^{H}}{B}\right]=2 A \frac{J_{0}}{E\left[B^{2}\right]}
$$

proportional to the risk aversion $A$ and the intrinsic financial risk $J_{0}$ of $H$. Finally, note that the valuation in (3.4) does not depend on the initial capital $c$ and is not linear in the number $\gamma$ of claims. It might be interesting to compare this to empirically observed prices.

Remark. Let us pause here for a moment to comment on the interpretation of $h_{1}$. Despite our terminology, we do not claim that $h_{1}(\gamma)$ is necessarily "the price for $\gamma$ units of $H$ "; it is rather a possible value assigned to $\gamma$ units of $H$ by someone who is a priori willing to use $u_{1}$ as valuation and then to accept the above indifference argument. One can also think of $h_{1}(\gamma)$ as a benchmark value against which one can compare one's own subjective assessments. In particular, the fact that $h_{1}(z \gamma) \neq z h_{1}(\gamma)$ does not entail arbitrage opportunities because it may not be possible to actually trade at the "prices" suggested by $h_{1}$.

\section{The financial standard deviation principle}

In this section, we determine the $u$-indifference prices $h_{2}(c, \gamma)$ for the valuation principle

$$
u_{2}(Y):=E^{B}\left[\frac{Y}{B}\right]-A \sqrt{\operatorname{Var}^{B}\left[\frac{Y}{B}\right]} .
$$

By repeating the arguments in the proof of Theorem 9, we get

$$
\begin{aligned}
h_{2}(\gamma)=\gamma c^{H} & +\sup _{g \in \overline{\mathcal{G}}}\left(E^{B}\left[\frac{g}{B}\right]-A \sqrt{\operatorname{Var}^{B}\left[\frac{g}{B}\right]}\right) \\
& -\sup _{g^{\prime} \in \overline{\mathcal{G}}}\left(E^{B}\left[\frac{g^{\prime}}{B}\right]-A \sqrt{\operatorname{Var}^{B}\left[\frac{g^{\prime}}{B}\right]+\gamma^{2} \operatorname{Var}^{B}\left[\frac{N^{H}}{B}\right]}\right) .
\end{aligned}
$$


We start by analyzing the last term.

Lemma 10. Let $\mathcal{G}$ be a linear subspace of $L^{2}$ admitting no approximate profits in $L^{2}$. For any $y \in \mathbb{R}$, we then have

$$
\begin{aligned}
& \sup _{g \in \overline{\mathcal{G}}}\left(E^{B}\left[\frac{g}{B}\right]-A \sqrt{\operatorname{Var}^{B}\left[\frac{g}{B}\right]+y^{2}}\right) \\
& = \begin{cases}-A \sqrt{y^{2}} & \text { for } B \in \mathcal{G}^{\perp} \\
\sup _{m \in \mathbb{R}}\left(m-A \sqrt{\frac{m^{2}}{\operatorname{Var}^{B}\left[\frac{d \widetilde{P}}{d P^{B}}\right]}+y^{2}}\right) & \text { for } B \notin \mathcal{G}^{\perp} .\end{cases}
\end{aligned}
$$

Proof. If $B \in \mathcal{G}^{\perp}$, then $E^{B}\left[\frac{g}{B}\right]=\frac{1}{E\left[B^{2}\right]} E[B g]=0$ for all $g \in \overline{\mathcal{G}}$ and so we can simply minimize the $P^{B}$-variance of $\frac{g}{B}$ by choosing $g=0$. For the case where $B \notin \mathcal{G}^{\perp}$, the idea is to perform the maximization in two steps by first restricting attention to those $g$ satisfying the constraint $E^{B}\left[\frac{g}{B}\right]=m$. In analogy to Corollary 16 of Schweizer (1996), we therefore define

$$
g_{m}:=c_{m}(B-\pi(B)):=\frac{m E\left[B^{2}\right]}{E\left[(B-\pi(B))^{2}\right]}(B-\pi(B))
$$

Since $B \notin \mathcal{G}^{\perp}$, we have $E[B(B-\pi(B))]=E\left[(B-\pi(B))^{2}\right]>0$ and so $g_{m}$ is well-defined and in $\mathcal{G}^{\perp \perp}=\overline{\mathcal{G}}$ since $\mathcal{G}$ is linear. Moreover,

$$
E^{B}\left[\frac{g_{m}}{B}\right]=\frac{1}{E\left[B^{2}\right]} E\left[B g_{m}\right]=m .
$$

If we take any $g \in \overline{\mathcal{G}}$ with $E^{B}\left[\frac{g}{B}\right]=m$, then

$$
\begin{aligned}
\left\|g-c_{m} B\right\|^{2} & =\left\|g-g_{m}+c_{m} \pi(B)\right\|^{2} \\
& =\left\|g-g_{m}\right\|^{2}+c_{m}^{2}\|\pi(B)\|^{2} \\
& \geq c_{m}^{2}\|\pi(B)\|^{2} \\
& =\left\|g_{m}-c_{m} B\right\|^{2}
\end{aligned}
$$

and so we deduce that

$$
\operatorname{Var}^{B}\left[\frac{g}{B}\right]=\operatorname{Var}^{B}\left[\frac{g}{B}-c_{m}\right]=\frac{1}{E\left[B^{2}\right]}\left\|g-c_{m} B\right\|^{2}-\left(m-c_{m}\right)^{2} \geq \operatorname{Var}^{B}\left[\frac{g_{m}}{B}\right]
$$


This implies that

$$
\begin{aligned}
& \sup \left\{E^{B}\left[\frac{g}{B}\right]-A \sqrt{\operatorname{Var}^{B}\left[\frac{g}{B}\right]+y^{2}} \mid g \in \overline{\mathcal{G}} \text { with } E^{B}\left[\frac{g}{B}\right]=m\right\} \\
& =E^{B}\left[\frac{g_{m}}{B}\right]-A \sqrt{\operatorname{Var}^{B}\left[\frac{g_{m}}{B}\right]+y^{2}} \\
& =m-A \sqrt{c_{m}^{2} \operatorname{Var}^{B}\left[1-\frac{\pi(B)}{B}\right]+y^{2}} .
\end{aligned}
$$

But

$$
\begin{aligned}
\operatorname{Var}^{B}\left[1-\frac{\pi(B)}{B}\right] & =\frac{1}{E\left[B^{2}\right]}\|B-\pi(B)\|^{2}-\left(\frac{1}{E\left[B^{2}\right]} E[B(B-\pi(B))]\right)^{2} \\
& =\frac{1}{E\left[B^{2}\right]}\|B-\pi(B)\|^{2}-\left(\frac{1}{E\left[B^{2}\right]} E\left[(B-\pi(B))^{2}\right]\right)^{2}
\end{aligned}
$$

and so we get

$$
c_{m}^{2} \operatorname{Var}^{B}\left[1-\frac{\pi(B)}{B}\right]=m^{2}\left(\frac{E\left[B^{2}\right]}{E\left[(B-\pi(B))^{2}\right]}-1\right)=m^{2} \frac{\|\pi(B)\|^{2}}{\|B-\pi(B)\|^{2}} .
$$

Together with (2.4), this proves the assertion.

q.e.d.

The next result is elementary analysis; its proof is only included for completeness.

Lemma 11. For any $y \in \mathbb{R}$, let

$$
s(y):=\sup _{x \in \mathbb{R}}\left(x-\sqrt{C x^{2}+y^{2}}\right)
$$

for a fixed $C \geq 0$. Then

$$
s(0)-s(y)= \begin{cases}\sqrt{y^{2}} \sqrt{1-\frac{1}{C}} & \text { for } C \geq 1 \\ \text { undefined } & \text { for } C<1\end{cases}
$$

Proof. Fix $y \in \mathbb{R}$ and let $f(x):=x-\sqrt{C x^{2}+y^{2}}$ for $x \in \mathbb{R}$. Then

$$
\lim _{x \rightarrow \pm \infty} \frac{f(x)}{|x|}=\lim _{x \rightarrow \pm \infty}\left( \pm 1-\sqrt{C+\frac{y^{2}}{x^{2}}}\right)= \pm 1-\sqrt{C}
$$


For $C<1$, this implies that $\lim _{x \rightarrow \pm \infty} f(x)= \pm \infty$ and since $f$ is continuous, we conclude that $f$ has no finite maximum so that $s \equiv+\infty$ in this case. For $C \geq 1$, we write $f(x)$ as

$$
f(x)=\frac{x^{2}-\left(C x^{2}+y^{2}\right)}{x+\sqrt{C x^{2}+y^{2}}}=\frac{(1-C) x^{2}-y^{2}}{x+\sqrt{C x^{2}+y^{2}}}
$$

this shows that $f \leq 0$. If $C=1$, the numerator does not depend on $x$ and the denominator goes to $+\infty$ for $x \rightarrow+\infty$. Hence we conclude that $s \equiv 0$ in this case. If $C>1$ and $y=0$, the maximum of $f \leq 0$ is attained in $x=0$ so that $s(0)=0$ for $C>1$. Finally, if $C>1$ and $y^{2}>0, f$ is continuously differentiable with derivative $f^{\prime}(x)=1-\frac{C x}{\sqrt{C x^{2}+y^{2}}}$. Since $f^{\prime}>0$ for $x \leq 0$, it is easily checked that $f^{\prime}$ vanishes at the unique point $x^{*}=\sqrt{\frac{y^{2}}{C(C-1)}}$ and $f\left(x^{*}\right)=-\sqrt{y^{2}} \sqrt{1-\frac{1}{C}}$ by computation. By (4.4), $f$ must have its maximum at $x^{*}$ and so the assertion follows.

q.e.d.

Combining the two previous results, we now obtain

Theorem 12. Let $\mathcal{G}$ be a linear subspace of $L^{2}$ admitting no approximate profits in $L^{2}$. For any $H \in L^{2}$ and any $\gamma, c \in \mathbb{R}$, the $u_{2}$-indifference price for $\gamma$ units of $H$ is then

$$
\begin{aligned}
h_{2}(c, \gamma) & =h_{2}(\gamma) \\
& = \begin{cases}\gamma \widetilde{E}\left[\frac{H}{B}\right]+A|\gamma| \sqrt{1-\frac{\operatorname{Var}^{B}\left[\frac{d \widetilde{P}}{d P^{B}}\right]}{A^{2}} \sqrt{\operatorname{Var}^{B}\left[\frac{N^{H}}{B}\right]}} & \text { for } A^{2} \geq \operatorname{Var}^{B}\left[\frac{d \widetilde{P}}{d P^{B}}\right] \\
\text { undefined } & \text { for } A^{2}<\operatorname{Var}^{B}\left[\frac{d \widetilde{P}}{d P^{B}}\right],\end{cases}
\end{aligned}
$$

where $\widetilde{E}$ denotes expectation with respect to the $B$-variance-optimal signed $(\mathcal{G}, B)$-martingale measure $\widetilde{P}$.

Proof. We first observe that if $B \in \mathcal{G}^{\perp}$, then $\pi(B)=B$ and therefore $\operatorname{Var}^{B}\left[\frac{d \widetilde{P}}{d P^{B}}\right]=0$ by (2.2). This shows that for $B \in \mathcal{G}^{\perp}$, the second case in (4.5) will never occur. If $B \in \mathcal{G}^{\perp}$, then (4.2) and (4.3) imply that

$$
h_{2}(\gamma)=\gamma c^{H}+A \sqrt{\gamma^{2} \operatorname{Var}^{B}\left[\frac{N^{H}}{B}\right]}=\gamma \widetilde{E}\left[\frac{H}{B}\right]+A|\gamma| \sqrt{\operatorname{Var}^{B}\left[\frac{N^{H}}{B}\right]}
$$

by Corollary 7. If $B \notin \mathcal{G}^{\perp}$, then (4.2) and (4.3) yield

$$
h_{2}(\gamma)=\gamma c^{H}+\sup _{m \in \mathbb{R}}\left(m-\sqrt{C m^{2}}\right)-\sup _{m \in \mathbb{R}}\left(m-\sqrt{C m^{2}+A^{2} \gamma^{2} \operatorname{Var}^{B}\left[\frac{N^{H}}{B}\right]}\right)
$$


where we have set

$$
C:=\frac{A^{2}}{\operatorname{Var}^{B}\left[\frac{d \widetilde{P}}{d P^{B}}\right]} \geq 0
$$

From Lemma 11 and Corollary 7, we thus obtain

$$
h_{2}(\gamma)=\gamma \widetilde{E}\left[\frac{H}{B}\right]+A|\gamma| \sqrt{1-\frac{\operatorname{Var}^{B}\left[\frac{d \widetilde{P}}{d P^{B}}\right]}{A^{2}}} \sqrt{\operatorname{Var}^{B}\left[\frac{N^{H}}{B}\right]}
$$

for $C \geq 1$, while $h_{2}(\gamma)$ is undefined for $C<1$. This proves the assertion.

q.e.d.

Like (3.4), the valuation formula (4.5) has a very appealing interpretation. If we write

$$
\pm h_{2}( \pm 1)= \begin{cases}\widetilde{E}\left[\frac{H}{B}\right] \pm A \sqrt{1-\frac{\operatorname{Var}^{B}\left[\frac{d \widetilde{P}}{d P^{B}}\right]}{A^{2}} \sqrt{\operatorname{Var}^{B}\left[\frac{N^{H}}{B}\right]}} & \text { for } A^{2} \geq \operatorname{Var}^{B}\left[\frac{d \widetilde{P}}{d P^{B}}\right] \\ \text { undefined } & \text { for } A^{2}<\operatorname{Var}^{B}\left[\frac{d \widetilde{P}}{d P^{B}}\right]\end{cases}
$$

we see that our approach transforms the actuarial standard deviation principle (4.1) into the financial standard deviation principle (4.6). Like (3.4), the valuation in (4.5) is based on the expectation under the $B$-variance-optimal signed $(\mathcal{G}, B)$-martingale measure $\widetilde{P}$ and the intrinsic financial risk of $H$. The corresponding bid-ask spread is given by

$$
h_{2}(+1)+h_{2}(-1)= \begin{cases}2 A \sqrt{1-\frac{\operatorname{Var}^{B}\left[\frac{d \widetilde{P}}{d P^{B}}\right]}{A^{2}} \sqrt{\operatorname{Var}^{B}\left[\frac{N^{H}}{B}\right]}} & \text { for } A^{2} \geq \operatorname{Var}^{B}\left[\frac{d \widetilde{P}}{d P^{B}}\right] \\ \text { undefined } & \text { for } A^{2}<\operatorname{Var}^{B}\left[\frac{d \widetilde{P}}{d P^{B}}\right]\end{cases}
$$

In contrast to (3.4), the valuation (4.5) is piecewise linear in the number $\gamma$ of claims; hence the resulting selling and buying "prices" for an arbitrary amount of $H$ are proportional to the selling and buying "price" of 1 unit of $H$ respectively. A second major difference to the last section is that all these results require a sufficiently high risk aversion for $h_{2}(c, \gamma)$ to be well-defined. The lower bound on $A$ depends on the $P^{B}$-variance of the density of $\widetilde{P}$ with respect to $P^{B}$. It is thus determined by the global properties of the financial environment $(\mathcal{G}, B)$ and in particular independent of the individual claim under consideration. In a very special case, a result like Theorem 12 has also been obtained by Aurell/Życzkowski (1996) by means of rather laborious calculations. 


\section{Two basic examples and an extension}

In this section, we briefly discuss the two extreme cases of an inexistent and a complete financial market and mention an extension of our approach to take into account already existing positions. For reasons of space, explicit examples for the environment $(\mathcal{G}, B)$ that allow for more concrete computations with the resulting financial valuation rule will be discussed elsewhere. We refer to Mercurio (1996), Aurell/Życzkowski (1996) and Gharagozlou (1997) for some preliminary studies and to Møller (1999, 2000) for applications in an actuarial context.

\subsection{The case of no financial market}

We first consider the case $\mathcal{G}=\{0\}$; this describes a situation without a financial market because every self-financing trading strategy has outcome 0. Since there is nothing to optimize, the general indifference condition (3.1) reduces to

$$
u(c B)=u(c B+h(c, \gamma) B-\gamma H)
$$

this is very similar to the principle of zero utility as in Gerber (1979). For $u \in\left\{u_{1}, u_{2}\right\}$, we obtain as in Lemma 8

$$
h_{1,2}(c, \gamma)=h_{1,2}(\gamma)=-u_{1,2}(-\gamma H)=\gamma E^{B}\left[\frac{H}{B}\right]+A\left(\gamma^{2} \operatorname{Var}^{B}\left[\frac{H}{B}\right]\right)^{\beta_{1,2}}
$$

so that the actuarial and financial valuation principles coincide in this case. Moreover, $\mathcal{G}^{\perp}=L^{2}$ implies that $\pi$ is the identity mapping, hence $\pi(B)=B$, and so comparing $(2.2)$ and (2.1) shows that $\widetilde{P}=P^{B}$, i.e., the original measure $P^{B}$ is already variance-optimal.

\subsection{The case of a complete financial market}

The other extreme case occurs if $(\mathcal{G}, B)$ describes a complete financial market where every claim $H \in L^{2}$ is attainable. Note that this does not mean that $\mathcal{G}=L^{2}$ because then we would have $B \in \mathcal{G}$ and therefore arbitrage. The correct description is that $B$ is not in $\overline{\mathcal{G}}$ (so that $\mathcal{G}$ admits no approximate profits in $L^{2}$ ) and that $\overline{\mathcal{A}}=\mathbb{R} B+\overline{\mathcal{G}}=L^{2}$. By Corollary 3 , the non-hedgeable part $N^{H}$ of every claim $H \in L^{2}$ is then 0 , and so (3.1) takes the form

$$
\sup _{g \in \mathcal{G}} u(c B+g)=\sup _{g \in \mathcal{G}} u\left(c B+\left(h(c, \gamma)-\gamma c^{H}\right) B+g-\gamma g^{H}\right)
$$

Of course, the mapping $g \mapsto g^{\prime}:=g-\gamma g^{H}$ is a bijection of $\overline{\mathcal{G}}$ into itself. If we have

$$
\sup _{g \in \mathcal{G}} u(g+Y)=\sup _{g \in \overline{\mathcal{G}}} u(g+Y)
$$


for every $Y \in L^{2}$, we can therefore conclude that

$$
h(c, \gamma)=h(\gamma)=\gamma c^{H}=\gamma \widetilde{E}\left[\frac{H}{B}\right]
$$

is a $u$-indifference price; this slightly generalizes the remark in section 3 . Condition (5.1) is trivially satisfied for any choice of $u$ if $\mathcal{G}$ is closed in $L^{2}$. Without this assumption, (5.1) still holds for $u \in\left\{u_{1}, u_{2}\right\}$; see the proof of Lemma 8 .

\subsection{Extension to an initial position}

Up to now, we have assumed that we start at time 0 with just an initial capital of $c$ and then value a claim $H$ on that basis. But in more realistic situations, one has to examine cases where one already has some position (a book of options or an insurance portfolio) and is adding a new claim $H$ on top of that. If we describe the existing position by its final time payoff $H_{0} \in L^{2}$, we have to replace the value functions of the optimization problems (i) and (ii) in section 3 by

$$
v\left(c, 0, H, 0 ; H_{0}\right):=\sup _{g \in \mathcal{G}} u\left(c B-H_{0}+g\right)
$$

and

$$
v\left(c, \gamma, H, x ; H_{0}\right):=\sup _{g \in \mathcal{G}} u\left((c+x) B-H_{0}+g-\gamma H\right) .
$$

In words, $v\left(c, \gamma, H, x ; H_{0}\right)$ is the maximal subjective value achievable by trading in $\mathcal{G}$ if one has initial capital $c$ and position $H_{0}$ and sells $\gamma$ units of $H$ for an amount of $x$. The condition (3.1) for the utility indifference price $h\left(c, \gamma, H ; H_{0}\right)$ then becomes

$$
v\left(c, \gamma, H, h\left(c, \gamma, H ; H_{0}\right) ; H_{0}\right)=v\left(c, 0, H, 0 ; H_{0}\right) .
$$

Essentially the same arguments as in section 3 lead to

$$
\begin{aligned}
h_{1}\left(c, \gamma, H ; H_{0}\right) & =h_{1}\left(\gamma, H ; H_{0}\right) \\
& =\gamma \widetilde{E}\left[\frac{H}{B}\right]+A \gamma^{2} \operatorname{Var}^{B}\left[\frac{N^{H}}{B}\right]+2 A \gamma \operatorname{Cov}^{B}\left(\frac{N^{H}}{B}, \frac{H_{0}}{B}\right) \\
& =\gamma \widetilde{E}\left[\frac{H}{B}\right]+A \gamma^{2} \operatorname{Var}^{B}\left[\frac{N^{H}}{B}\right]+2 A \gamma \operatorname{Cov}^{B}\left(\frac{N^{H}}{B}, \frac{N^{H_{0}}}{B}\right)
\end{aligned}
$$

and so the valuation contains an additional term to account for the correlation between the existing position $H_{0}$ and the non-hedgeable part $N^{H}$ of the new claim $H$. Note that as for the variance component, the attainable part $g^{H}$ does not enter; the same is true for $g^{H_{0}}$ since $\operatorname{Cov}^{B}\left(\frac{N^{H}}{B}, \frac{g^{H_{0}}}{B}\right)=0$ by Lemma 5 . If we repeat the arguments of section 4 , we also find (5.4) $h_{2}\left(c, \gamma, H ; H_{0}\right)=h_{2}\left(\gamma, H ; H_{0}\right)$

$$
=\gamma \widetilde{E}\left[\frac{H}{B}\right]+A \sqrt{1-\frac{\operatorname{Var}^{B}\left[\frac{d \widetilde{P}}{d P^{B}}\right]}{A^{2}}}\left(\sqrt{\operatorname{Var}^{B}\left[\frac{N^{H_{0}}+\gamma N^{H}}{B}\right]}-\sqrt{\operatorname{Var}^{B}\left[\frac{N^{H_{0}}}{B}\right]}\right)
$$


for $A^{2} \geq \operatorname{Var}^{B}\left[\frac{d \widetilde{P}}{d P^{B}}\right]$, whereas $h_{2}\left(c, \gamma, H ; H_{0}\right)$ is again undefined for $A^{2}<\operatorname{Var}^{B}\left[\frac{d \widetilde{P}}{d P^{B}}\right]$.

As an application of the preceding extension, we can answer the following question raised by D. Lando: What happens if one splits a payoff $H$ into the sum of two components $H_{1}, H_{2}$ and values these components separately? If we use the original approach in (3.1), it may well happen that the sum of the resulting prices for $H_{1}$ and $H_{2}$ is strictly larger than the price for the sum $H=H_{1}+H_{2}$. Consider for instance a purely financial attainable payoff $H$ and split $H$ into $H_{1}=H I_{D}$ and $H_{2}=H I_{D^{c}}$ where $D$ is the event that a specified person dies before a specified date. Both $H_{1}$ and $H_{2}$ are then so-called unit-linked life insurance products; the above person may want to purchase $\mathrm{H}_{2}$ as part of a pension plan while his/her partner or heirs may be more interested in $H_{1}$. (I am grateful to D. Lando for this colourful example.) Then both Theorem 9 and Theorem 12, applied separately to $H_{1}$ and $H_{2}$, would charge in total more than the arbitrage-free price of $H$ which clearly should not happen.

The flaw in the above reasoning is of course that one does not take into account the changed position once $H_{1}$ or $H_{2}$ has been sold. Once this is remedied by appropriately using (5.2) instead of (3.1), we obtain in fact the very natural result that

$$
h\left(c, \gamma, H_{1}+H_{2} ; H_{0}\right)=h\left(c, \gamma, H_{1} ; H_{0}\right)+h\left(c+h\left(c, \gamma, H_{1} ; H_{0}\right), \gamma, H_{2} ; H_{0}+\gamma H_{1}\right) .
$$

Put into words, this says that the price for selling directly $\gamma$ units of $H_{1}+H_{2}$ equals the sum of the prices for selling first $\gamma$ units of $H_{1}$ and then $\gamma$ units of $H_{2}$ if the second sale takes into account that the first sale has changed both the initial capital (from $c$ to $c+h\left(c, \gamma, H_{1} ; H_{0}\right)$ ) and the position (from $H_{0}$ to $H_{0}+\gamma H_{1}$ ).

To see why (5.5) is true, we set $x_{1}:=h\left(c, \gamma, H_{1} ; H_{0}\right), x_{2}:=h\left(c+x_{1}, \gamma, H_{2} ; H_{0}+\gamma H_{1}\right)$ and $x:=h\left(c, \gamma, H_{1}+H_{2} ; H_{0}\right)$ so that we have to show that $x=x_{1}+x_{2}$. Since clearly

$$
v(y, 0, H, 0 ; J)=v\left(y, 0, H^{\prime}, 0 ; J\right)
$$

for any choice of $y, H, H^{\prime}, J$, using repeatedly (5.2) and the definition of $v$ gives

$$
\begin{aligned}
v\left(c, \gamma, H_{1}+H_{2}, x ; H_{0}\right) & =v\left(c, 0, H_{1}+H_{2}, 0 ; H_{0}\right) \\
& =v\left(c, 0, H_{1}, 0 ; H_{0}\right) \\
& =v\left(c, \gamma, H_{1}, x_{1} ; H_{0}\right) \\
& =\sup _{g \in \mathcal{G}} u\left(\left(c+x_{1}\right) B-\left(H_{0}+\gamma H_{1}\right)+g\right) \\
& =v\left(c+x_{1}, 0, H_{2}, 0 ; H_{0}+\gamma H_{1}\right) \\
& =v\left(c+x_{1}, \gamma, H_{2}, x_{2} ; H_{0}+\gamma H_{1}\right) \\
& =\sup _{g \in \mathcal{G}} u\left(\left(c+x_{1}+x_{2}\right) B-H_{0}+g-\gamma\left(H_{1}+H_{2}\right)\right) \\
& =v\left(c, \gamma, H_{1}+H_{2}, x_{1}+x_{2} ; H_{0}\right) .
\end{aligned}
$$


Under the mild assumption that (5.2) uniquely determines $h\left(c, \gamma, H ; H_{0}\right)$, we deduce (5.5). For the special cases of $u_{1}, u_{2}$, this could also be obtained by direct computations from (5.3) or $(5.4)$.

\section{References}

E. Aurell and K. Życzkowski (1996), "Option Pricing \& Partial Hedging: Theory of Polish Options", preprint, Royal Institute of Technology, Stockholm

H. Bühlmann (1987), "Actuaries of the Third Kind?", ASTIN Bulletin 17, 137-138

H. Bühlmann (1997), "The Actuary: The Role and Limitations of the Profession since the Mid-19th Century", ASTIN Bulletin 27, 165-171

T. Choulli, L. Krawczyk and C. Stricker (1998), "E-Martingales and their Applications in Mathematical Finance", Annals of Probability 26, 853-876

T. Choulli, L. Krawczyk and C. Stricker (1999), "On Fefferman and Burkholder-DavisGundy Inequalities for $\mathcal{E}$-Martingales", Probability Theory and Related Fields 113, 571-597

F. Delbaen, P. Monat, W. Schachermayer, M. Schweizer and C. Stricker (1997), "Weighted Norm Inequalities and Hedging in Incomplete Markets", Finance and Stochastics 1, 181-227

F. Delbaen and W. Schachermayer (1996), "The Variance-Optimal Martingale Measure for Continuous Processes", BERNOULLI 2, 81-105; Amendments and corrections (1996), BERNOULLI 2, 379-380

H. U. Gerber (1979), "An Introduction to Mathematical Risk Theory", Huebner Foundation Monograph No. 8, Irwin, Illinois

A. Gharagozlou (1997), "Zur Bewertung von Optionen mit einer mean-variance-Nutzenfunktion", diploma thesis, Technical University of Berlin

D. Kliger and B. Levikson (1998), "Pricing Insurance Contracts - an Economic Viewpoint", Insurance: Mathematics and Economics 22, 243-249

F. Mercurio (1996), "Variance-Optimal Hedging and Risk Preferences", preprint, Erasmus University Rotterdam

T. Møller (1999), "On Indifference Pricing for Insurance Contracts", preprint, Laboratory of Actuarial Mathematics, University of Copenhagen

T. Møller (2000), "On Transformations of Actuarial Valuation Principles", Proceedings 
of the 10th AFIR Colloquium, Tromsø, 517-545

M. Schweizer (1995), "Variance-Optimal Hedging in Discrete Time", Mathematics of Operations Research 20,1-32

M. Schweizer (1996), "Approximation Pricing and the Variance-Optimal Martingale Measure", Annals of Probability 24, 206-236

M. Schweizer (1999), "Risky Options Simplified", International Journal of Theoretical and Applied Finance 2, 59-82

C. Stricker (1990), "Arbitrage et Lois de Martingale”, Annales de l'Institut Henri Poincaré $26,451-460$ 